\title{
Optoelectronic Device Based on Gunn Effect Extended Diode Structure
}

\author{
I. TRALLE* \\ Institute of Physics, University of Rzeszów \\ al. Rejtana 16A, 35-310 Rzeszów, Poland
}

(Received September 11, 2006)

\begin{abstract}
We discuss the possibility of light control by means of light diffraction by space charge waves which are the periodic domain trains induced at some circumstances in GaAs Gunn effect diode. The two possible regimes of the proposed device are considered: the first one which is analogous to the Bragg diffraction in case of light-acoustic diffraction and the other one, which we call "intermediate", since the parameter $Q$ which distinguishes the Raman-Nath $(Q<1)$ and the Bragg diffraction $(Q>10)$, in this case is $\approx 1$ (actually, only bit smaller than 1 ). Among the advantages of this hypothetical device are the simpler control of operation and perhaps, possibility to couple it with the waveguides and switchers for surface electromagnetic waves and other miniaturized photonic circuits.
\end{abstract}

PACS numbers: 42.25.Fx, 42.79.Dj, 42.79.Gn, 42.82.Et, 42.82.Fv

\section{Introduction}

So far in most of the light deflectors and directional couplers the acoustic surface waves were used in order to get modulation of the dielectric susceptibility. Nevertheless, the search for other possibilities of light control has never ceased (see, for example, the recent paper [1] and the references cited therein). We would like to attract attention to another possibility: the modulation, similar to that in case of surface acoustic waves, can be achieved also in thin semiconductor films by means of the induced space charge wave. The space charge wave (SCW) is nothing else but the domains of higher and lower charge densities alternating each other and moving with drift velocity. The well-known example of such SCWs are the plasmons induced in semiconductor plasma [2]. Another type of charge movement which looks like moving waves are the periodic domain trains induced at some circumstances in GaAs Gunn effect amplifier [3, 4].

*e-mail: tralle@univ.rzeszow.pl 
Since the dielectric susceptibility $\epsilon$ is proportional, to the first approximation, to the squared plasma frequency, it is clear that one can modulate $\epsilon$ by producing the moving domains of lower and higher charge densities, that is, by inducing the SCW. In this way one can make the light control possible due to light diffraction by SCW, just like it is achieved in deflectors and directional couplers based on surface acoustic waves.

In this paper we propose the model and discuss the theory of such device based on GaAs Gunn effect diode in which multiple domain regime is to occur. We consider light diffraction by modulation of semiconductor dielectric susceptibility caused by space charge wave and distinguish two regimes, the Bragg diffraction and another one, which is intermediate between the Bragg and the Raman-Nath diffraction.

\section{The model and basic equations of light diffraction by SCW}

Let us argue first that light diffraction by SCW is possible. The point is that sometimes one can encounter the statement that light wave cannot interact with plasmons [5], since plasma oscillations, just like the other types of SCW, are longitudinal, while electromagnetic waves are transversal. This statement, being correct for electromagnetic waves in free space, is no longer valid in case of waveguides where both types of waves, transverse electric (TE), as well as transverse magnetic (TM) can propagate. We assume two possibilities to occur: either the semiconductor sample in which SCWs are induced, is inserted into the waveguide where TM electromagnetic wave propagates (such wave by definition, has the component of electric field which is the projection of $\boldsymbol{E}$ vector onto the SCW wave vector and hence, such electromagnetic wave can interact with SCW), or the electromagnetic wave is incident on the sample at such an angle that there is a non-zero projection of $\boldsymbol{E}$ vector of the electromagnetic wave onto the plane in which the wave vector of SCW lies.

In most of the cases in the Gunn effect device only a single field domain is generated at a time and hence, this travelling domain hardly can be called "wave" or at least, harmonic-type wave. However, at some circumstances the multiple domains can also be generated $[3,4]$ and this operating regime to some approximation can be treated in terms of harmonic wave process.

Let us suppose we have distributed semiconductor structure in which moving multiple Gunn domains are generated and which is represented schematically in Fig. 1. "Distributed" in this context means that the sample length in $y$-direction $\left(L_{y}\right)$ is much greater than in $x$-direction $\left(L_{x}\right): L_{y} \gg L_{x}$. Let us notice that such distributed structures were already discussed in the literature [6], though in entirely different context. We assume that the SCW associated with periodic domains moves in $x$-direction and can be characterized by the wave vector $k_{0}$ and frequency $\Omega$. In the simplest case the carriers concentration accompanying 


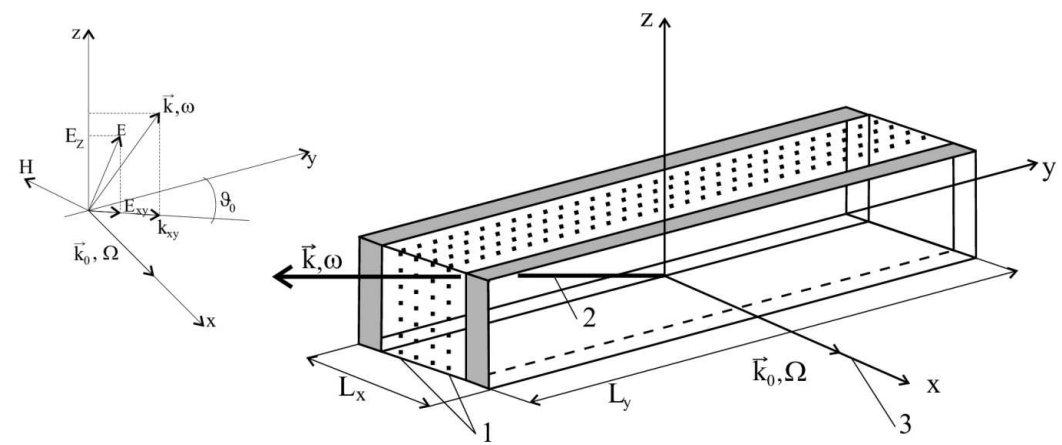

Fig. 1. Schematic representation of distributed Gunn diode structure: 1 - moving periodic high electric field domains accompanied by space charge wave are shown schematically; 2 - light beam incident on the semiconductor and characterized by $\boldsymbol{k}$ and $\omega$, 3 - space charge wave propagates along $x$-axis and is characterized by $\boldsymbol{k}_{0}$ and $\Omega$. In the inset the reference frame is depicted in greater scale; $\boldsymbol{E}$ and $\boldsymbol{H}$ components of TM electromagnetic wave are shown; $E_{x y}$ stands for the $\boldsymbol{E}$ vector projection onto $(x, y)$ plane; it makes the angle $\theta_{0}$ with $y$-axis.

this SCW can be represented as $n=n_{0}+n_{1} \cos \left(\Omega t-k_{0} x\right)$. Here $n_{0}$ is the equilibrium electron concentration (in the absence of the field domains) and $n_{1}$ is the excess concentration due to the high field domains. Clearly enough, the wave vector and the frequency are related by $k_{0}=\Omega / v_{\mathrm{d}}$, where $v_{\mathrm{d}}$ is the drift velocity. The real part of the semiconductor dielectric susceptibility for the optical frequencies can be estimated roughly as $\epsilon(\infty) \sim 1-\omega_{\mathrm{p}}^{2} / \omega^{2}$, where $\omega_{\mathrm{p}}$ is the plasma frequency. Since plasma frequency is proportional to $n^{1 / 2}$, it is clear that the modulation of space charge density will lead to the dielectric susceptibility modulation $\epsilon=\epsilon_{0}+\Delta \epsilon \cos \left(\Omega t-k_{0} x\right)$. If the electromagnetic wave is incident on the semiconductor layer at some angle to the $y$-axis, it can be diffracted by this modulation wave just as it occurs in the case of light diffraction by ultrasonic vibrations. However, there is also an essential difference between diffraction by space charge modulation and that caused by acoustic wave. At the analysis of light diffraction by ultrasonic vibrations, one usually assumes the free charge carriers to be absent in the dielectric and hence the density of charge carriers $(n)$ and currents $(j)$ are supposed to be zero. Curiously enough, in metal optics, where neither $n$ nor $j$ are equal to zero, they are supposed to be zero, too. The point is that any initial perturbation of space charge $n_{0}$ decays exponentially with respect to time as $n(t)=n_{0} \exp \left(-t / \tau_{\mathrm{d}}\right.$ ), where $\tau_{\mathrm{d}}=\epsilon / \sigma$ is termed dielectric (or Maxwell) relaxation time, $\sigma$ is the sample conductivity. Since metal conductivity is very high, dielectric relaxation time is much less than the characteristic time of the changes of field amplitude for optical frequencies. Indeed, in metals $\tau_{\mathrm{d}}$ is about $10^{-18}-10^{-19} \mathrm{~s}$, that is, much smaller than $2 \pi / \omega \sim 10^{-15} \mathrm{~s}$ for optical frequencies. The last one makes it possible to assume $n$ and $j$ to be equal to zero, too. But in 
semiconductors the situation is quite different: neither $n$ nor $j$ are equal to zero and $\tau_{\mathrm{d}}$ is about $10^{-12} \mathrm{~s}$, which is greater than $2 \pi / \omega$ for optical frequencies. It would be logically unfair also to assume $n$ equal to zero, since the modulation of dielectric susceptibility is just determined by the space charge modulation. It is impossible therefore in our case (at least, without preliminary analysis) to suppose the charge and current densities to be zero in the initial Maxwell equations.

Let the plane linearly polarized electromagnetic wave $\boldsymbol{E}=\boldsymbol{E}_{0} \exp (\mathrm{i}(\omega t-\boldsymbol{k} \boldsymbol{r}))$ be incident on the semiconductor layer of the structure in question. Let us suppose $\boldsymbol{k}=\left(\boldsymbol{k}_{\mathrm{xy}}, k_{z}\right)$ where $\boldsymbol{k}_{x y}=\left(k_{x}, k_{y}\right)$. Let us also suppose that $\boldsymbol{k}_{\mathrm{xy}}$ makes an angle $\theta_{0}$ with $y$-axis as it is shown in Fig. 1 . Also let us assume $\boldsymbol{E}_{0}=\left(\boldsymbol{E}_{0 x y}, E_{0 z}\right)$ where $E_{0 z}$ is perpendicular to the $x y$-plane. Since we suppose the density of charge carriers to be independent of $y$ - and $z$-space variables, we can consider only $x$-component of electromagnetic field, $E_{x}=E_{0 x} \exp \left(\mathrm{i}\left(\omega t-k \sin \theta_{0} x\right)\right)$. As a result, we have to consider the Maxwell equations of the form (from now on we drop for simplicity the indices $x$ and $x y$ altogether):

$$
\begin{aligned}
& \nabla \times \boldsymbol{E}=-c^{-1} \frac{\partial \boldsymbol{B}}{\partial t} \cdot \nabla \boldsymbol{B}=\mathbf{0} . \\
& \nabla \times \boldsymbol{B}=c^{-1} \frac{\partial \boldsymbol{D}}{\partial t}+\frac{4 \pi}{c} \boldsymbol{j}, \quad \nabla \boldsymbol{D}=4 \pi \rho .
\end{aligned}
$$

where, as usual, $\rho$ and $j$ are the charge concentration and current density, respectively, $\rho=e n$, and $n$ stands for the electron concentration.

Some comments have to be added here: in the subsequent analysis we suppose that the frequency of the space charge wave, $\Omega$, is not greater than a few MHz. Therefore, the electrons are supposed to be able to follow the changes of the field amplitude. On the other hand, since the semiconductor conductivity is much smaller than the metal conductivity and drift velocity is much smaller than light velocity, we can assume the term $4 \pi j / c$ to be equal to zero in the Maxwell equations, incorporating the directed charge displacement in the form of travelling wave by means of $\rho=\rho\left(\Omega t-k_{0} x\right)$. In this way one can satisfy the charge conservation law $\partial \rho / \partial t+\nabla \boldsymbol{j}=0$, because the condition $4 \pi j / c \approx 0$ does not necessarily imply $\nabla \boldsymbol{j}=0$.

Using the standard procedure for deriving the wave equation and taking into account that $\Delta \epsilon \ll \epsilon_{0}$ and $\lambda / \Lambda<1$ ( $\lambda$ and $\Lambda$ are the wavelengths of the incident light and that of the SCW, respectively), we get

$$
\frac{\partial^{2} E}{\partial x^{2}}+\frac{\partial^{2} E}{\partial y^{2}}-\frac{1}{c^{\prime 2}} \frac{\partial^{2}}{\partial t^{2}}\left(1+\frac{\Delta \epsilon}{\epsilon_{0}}\right)=\frac{4 \pi}{\epsilon_{0}^{2}} \rho \nabla \epsilon-\frac{4 \pi}{\epsilon_{0}} \nabla \rho \equiv \mathcal{F}\left(\epsilon_{0}, \epsilon, \rho\right),
$$

where $c^{\prime}=c / \sqrt{\epsilon}$.

Let us search for the solution to (1) in the form of a series of plane waves corresponding to the different diffraction orders [7]: 


$$
E(x, y, t)=\sum_{n=-\infty}^{\infty} V_{n}(y) \exp \left(\mathrm{i}\left[(\omega+n \Omega) t-\left(k \sin \theta_{0}+n k_{0}\right) x-k y \cos \theta_{0}\right]\right) .
$$

Here $V_{n}(y)$ is the amplitude of the $n$-th diffraction beam of the frequency $\omega+n \Omega$. Using (2), after some manipulations (see Appendix) one arrives at the next equation for $V_{m}(y)$ :

$$
\begin{aligned}
\frac{\mathrm{d}^{2} V_{m}}{\mathrm{~d} y^{2}} & -2 \mathrm{i} \cos \theta_{0} \frac{\mathrm{d} V_{m}}{\mathrm{~d} y}+2 k \beta_{m} \cos \theta_{0} V_{m} \\
= & -\kappa\left(V_{m+1}+V_{m-1}\right)+\mathcal{F}_{m}\left(\epsilon_{0}, \Delta \epsilon, \rho\right) \exp \left(\mathrm{i} k \cos \theta_{0} y\right),
\end{aligned}
$$

where

$$
\begin{aligned}
& \beta_{m}=\left[2 m k k_{0}\left(v / c^{\prime}-\sin \theta_{0}\right)+m^{2} k_{0}^{2}\left(1-v^{2} / c^{\prime 2}\right)\right] / 2 k \cos \theta_{0}, \\
& \kappa=\frac{\Delta \epsilon}{2 \epsilon_{0}} k^{2}(1-m \Omega / \omega)^{2}, \quad \omega=c^{\prime} k, \quad \Omega=v_{\mathrm{d}} k_{0} .
\end{aligned}
$$

One can find the explicit formula for $\mathcal{F}_{m}$ in Appendix.

It is noteworthy that (3) is absolutely analogous to the corresponding equation describing the light diffraction by acoustic wave [8] and differs from the last one only by the term containing $\mathcal{F}_{m}$ in the right-hand side of Eq. (1), as it could be expected.

\section{Bragg diffraction by space charge wave}

Let us analyse at first the special case of the negligible amplitude of the second derivative $\mathrm{d}^{2} V_{m} / \mathrm{d} y^{2}$. Indeed, if $\Delta \epsilon / \epsilon_{0} \ll 1$, then $V_{m}$ varies relatively slow with respect to $y$ and $\mathrm{d}^{2} V_{m} / \mathrm{d} y^{2}$ is negligible compared to the other terms in $(3)$. As a result, we have the next equation to be analysed

$$
\begin{aligned}
\frac{\mathrm{d} V_{m}}{\mathrm{~d} y} & +\mathrm{i} \beta_{m} V_{m}=\frac{\mathrm{i}}{2 k \cos \theta_{0}}\left[\mathcal{F}_{m}\left(\Delta \epsilon, \epsilon_{0}, \rho_{1}, \rho_{0}\right) \exp \left(\mathrm{i} k \cos \theta_{0} y\right)\right. \\
& \left.-\left(\Delta \epsilon / \epsilon_{0}\right) k^{2}(1+m \Omega / \omega)^{2}\left(V_{m+1}+V_{m-1}\right)\right],
\end{aligned}
$$

where $k=\omega / c^{\prime}$. Supposing $m \Omega / \omega \ll 1$ (which is often the case), for small $m$ the solution to (4) can be expressed as

$$
\begin{aligned}
V_{m}(y) & =\left\{V_{m}\left(y_{0}\right)+\frac{\mathrm{i}}{2 k \cos \theta_{0}} \int_{y_{0}}^{y}\left[\mathcal { F } _ { m } \operatorname { e x p } \left(\mathrm{i} k \cos \theta_{0} y^{\prime}\right.\right.\right. \\
& \left.\left.-\frac{\Delta \epsilon}{\epsilon_{0}} k^{2}\left[V_{m+1}\left(y^{\prime}\right)+V_{m-1}\left(y^{\prime}\right)\right]\right] \exp \left(\mathrm{i} \beta_{m} y^{\prime}\right) \mathrm{d} y^{\prime}\right\} \exp \left(-\mathrm{i} \beta_{m} y\right),
\end{aligned}
$$

where $V_{m}\left(y_{0}\right)$ is the boundary value for the field amplitude of the $m$-th diffracted beam.

To compare the obtained result with the corresponding expression for the optical-acoustic diffraction, one has to make the same additional simplifications as in the theory of light diffraction by ultrasonic vibrations [8]. Let us assume therefore that not only the modulation of dielectric constant caused by space 
charge wave is small but that the $\theta_{0}$-angle is small, too. Formally this is reduced to the condition $\left|\int_{y_{0}}^{y} \Delta \epsilon k \mathrm{~d} y^{\prime} / \epsilon \cos \theta_{0}\right| \ll 1$. Then $V_{m+1} \ll V_{m}$ and hence we arrive at the formulae

$$
\begin{gathered}
V_{m}(y) \approx\left\{V_{m}\left(y_{0}\right)+\frac{\mathrm{i}}{2 k \cos \theta_{0}} \int_{y_{0}}^{y}\left[\mathcal{F}_{m} \exp \left(\mathrm{i} k \cos \theta_{0} y^{\prime}\right)\right.\right. \\
\left.\left.-\frac{\Delta \epsilon}{\epsilon_{0}} k^{2} V_{m-1}\left(y^{\prime}\right)\right] \exp \left(\mathrm{i} \beta_{m} y^{\prime}\right) \mathrm{d} y^{\prime}\right\} \exp \left(-\mathrm{i} \beta_{m} y\right),
\end{gathered}
$$

for $m \geq 0$, and

$$
\begin{gathered}
V_{m}(y) \approx\left\{V_{m}\left(y_{0}\right)+\frac{\mathrm{i}}{2 k \cos \theta_{0}} \int_{y_{0}}^{y}\left[\mathcal{F}_{m} \exp \left(\mathrm{i} k \cos \theta_{0} y^{\prime}\right)\right.\right. \\
\left.\left.-\frac{\Delta \epsilon}{\epsilon_{0}} k^{2} V_{m+1}\left(y^{\prime}\right)\right] \exp \left(\mathrm{i} \beta_{m} y^{\prime}\right) \mathrm{d} y^{\prime}\right\} \exp \left(-\mathrm{i} \beta_{m} y\right),
\end{gathered}
$$

for $m<0$.

Now let us make some estimates. Let us assume $\omega=1.5 \times 10^{15} \mathrm{~Hz}, \Omega=$ $6 \times 10^{9} \mathrm{~Hz}$ and the uniform equilibrium electron concentration in GaAs (in the absence of the space charge wave) to be $10^{14} \mathrm{~cm}^{-3}$. Let it be the peak value of electron concentration within the high field domains $\approx 10^{16} \mathrm{~cm}^{-3}$, which seems to be reasonable. Then the ratio $\Delta \epsilon / \epsilon_{0}$ is about $2.8 \times 10^{-6}$. Taking $v_{\mathrm{d}} \approx 10^{7} \mathrm{~cm} \mathrm{~s}^{-1}$, one has $\Lambda=2 \pi / k_{0} \approx 0.01047 \mathrm{~cm}$; so, the assumption which was made at the derivation of previous formulae seems to be justified. If we suppose the Bragg diffraction to occur and that only $V_{0}$ and $V_{ \pm 1}$ are nonzero just like in case of light diffraction by acoustic waves [7], then the quantity $\beta_{ \pm 1}$ can be represented as

$$
\beta_{ \pm 1}= \pm \frac{k_{0}}{\cos \theta_{0}}\left(\sin \alpha_{ \pm 1}-\sin \theta_{0}\right)
$$

where

$$
\sin \alpha_{ \pm 1}=v_{\mathrm{d}} / c^{\prime} \mp\left(k_{0} / 2 k\right)\left(1-v_{\mathrm{d}}^{2} / c^{\prime 2}\right) .
$$

If $v_{\mathrm{d}} / c^{\prime}$ were zero, $\alpha_{ \pm 1}$ would be exactly equal to the Bragg angle at the light diffraction by space charge wave. Since $v_{\mathrm{d}} / c^{\prime} \approx 0.0011$, the last relation can be rewritten as $\sin \alpha_{ \pm 1} \approx \sin \theta_{\mathrm{B}}=\Omega \Lambda /\left(2 v_{\mathrm{d}} \sqrt{\epsilon}\right)$, where $\theta_{\mathrm{B}}$ is the Bragg angle and $\lambda$ stands for the light wavelength.

Let us introduce, as in case of optical-acoustic diffraction, the "interaction length" $L$ corresponding to the light beam-acoustic wave interaction domain, so that $0 \leq y \leq L$ and let $y$ be in the $[-L / 2,+L / 2]$ interval. Let us assume also $\Delta \epsilon$ to be independent of $y$ inside the interval and equal to zero outside. Using (5) (or (6)), the formula for $\alpha_{ \pm 1}$ above and the standard boundary conditions $V_{0}\left(y_{0}\right)=V_{0}, V_{m}\left(y_{0}\right)=0$ for $m \neq 0$, the amplitude $V_{1}$ can be expressed as

$$
V_{1}=\frac{\mathrm{i}}{k \cos \theta_{0}} \exp \left(\mathrm{i} k_{0} y \frac{\sin \theta_{0}-\sin \alpha}{\cos \theta_{0}}\right) \frac{\sin \left(\frac{1}{2} L\left(\sin \theta_{0}-\sin \alpha\right) / \cos \theta_{0}\right)}{k_{0}\left(\sin \theta_{0}-\sin \alpha\right)}
$$




$$
\times\left(\mathcal{F}_{1}-\frac{\Delta \epsilon}{\epsilon_{0}} k^{2} V_{0}\right),
$$

where we dropped the \pm 1 subscripts of $\alpha$.

The relative intensity of the diffracted beams is of the form

$$
\left|V_{1} / V_{0}\right|^{2}=\left|\mathcal{F}_{1}-\frac{\Delta \epsilon}{\epsilon_{0}} k^{2} V_{0}\right|^{2} \frac{\sin ^{2}\left(\frac{1}{2} L\left(\sin \theta_{0}-\sin \alpha\right) / \cos \theta_{0}\right)}{k_{0}^{2}\left(\sin \theta_{0}-\sin \alpha\right)^{2}} .
$$

For $\mathcal{F}_{1}=0$ (that is, for $n, \rho$, and $\Delta \epsilon$ equal to zero) the last formula reduces exactly to the corresponding expression for optical-acoustic diffraction [8].

\section{4. "Intermediate" diffraction regime}

In the theory of light diffraction by ultrasonic waves, the parameter $Q=$ $2 \pi L \lambda / \sqrt{\epsilon} \Lambda^{2}$ characterizing diffraction, very often is used. It is commonly used to believe that if $Q>10$, the diffraction grating produces only one diffracted beam, while if $Q<1$, the grating produces many diffraction orders. The first case corresponds to the Bragg regime which in fact is the same as that considered in previous section, while the other one corresponds to the Raman-Nath regime.

The point however is that, first, drift velocity $v_{\mathrm{d}}$ usually is much greater than the sound velocity in a semiconductor and, second, $L$ in the formula for $Q$ for the distributed Gunn effect structure (remember, in our case $L \leq L_{y}$ ), in spite of the condition $L_{y} \gg L_{x}$, most probably cannot be greater than $1 \mathrm{~cm}$. As a result, the condition $Q>10$ hardly can be achieved in this case as well as the opposite one, $Q<1$. At the typical values of the parameters which enter the formula for $Q$, the last one is about $\approx 1$ (in fact, a bit smaller than unit). Taking these arguments into account, one should consider more general situation and analyse Eq. (4) and its solutions without an additional assumption that the amplitudes $V_{m}$ decrease rapidly with $m$ increasing.

Let us introduce new parameter $\zeta$ by means of $\zeta=\kappa / k \cos \theta_{0}$ and new variable $\chi$ by means of $\chi=2 \zeta y$. Then Eq. (4) can be rewritten as

$$
\begin{aligned}
2 V_{m}^{\prime} & +\left(V_{m+1}+V_{m-1}\right)=\left(\mathrm{i} \beta_{m} / \zeta\right) V_{m} \\
& +\left[\mathrm{i} \mathcal{F}_{m}\left(\epsilon_{0}, \epsilon_{1}, \rho\right) / \zeta k \cos \theta_{0}\right] \exp \left(\mathrm{i} k \cos \theta_{0} y\right) .
\end{aligned}
$$

And again, the difference between this equation and that one appearing in the theory of light-acoustic diffraction is only due to the term containing $\mathcal{F}_{m}$ in the right-hand side of (7). Following Raman and Nath, most of the people who dealt with this problem, set the term $\left(\mathrm{i} \beta_{m} / \zeta\right) V_{m}$ equal to zero in the equation above. In this way it is possible to reduce Eq. (7) to the recurrence relation for the Bessel functions of the first kind [9], since there is no term containing $\mathcal{F}_{m}$ in the right-hand side in case of light-acoustic diffraction and one can search for the real-valued solutions to this equation. However, as it was argued in Sect. 2, it would be logically inconsistent and incompatible with our approach to set $\mathcal{F}_{m}$ equal to zero. Hence, there is no much sense to assume $\left(\mathrm{i} \beta_{m} / \zeta\right) V_{m}$ term to be equal to zero, too. Now Eq. (7) with non-zero $\mathcal{F}_{m}$ and $\left(\mathrm{i} \beta_{m} / \zeta\right) V_{m}$ terms becomes 
complicated differential-recurrence equation which can be analysed numerically. It is beyond the scope of the present paper, however. Here instead, we carry out the subsequent analysis in somewhat different way.

Let us notice first that the functions $Z(x)$ defined by means of the recurrence relation

$$
Z_{m+1}-Z_{m-1}=-2 \frac{\mathrm{d} Z_{m}}{\mathrm{~d} x}
$$

are the Bessel functions which more frequently are defined as the solutions to the next differential equation [10]:

$$
x^{2} \frac{\mathrm{d}^{2} y}{\mathrm{~d} x^{2}}+x \frac{\mathrm{d} y}{\mathrm{~d} x}+\left(x^{2}-m^{2}\right) y=0 .
$$

Let us notice now that this equation is of the second order, and therefore it has two linearly independent solutions. When $m$ is an integer, the general (real) solution is of the form [10]:

$$
Z_{m} \equiv C_{1} J_{m}(x)+C_{2} Y_{m}(x),
$$

where $J_{m}$ is the Bessel function of the first kind, while $Y_{m}$ is the Bessel function of the second kind. The complex solutions to the equation above are given by the Hankel functions $H_{m}^{(1,2)}=J_{m}(z) \pm \mathrm{i} Y_{m}(z)$, where plus sign corresponds to $H_{m}^{(1)}$, the Hankel function of the first kind, while minus sign corresponds to the $H_{m}^{(2)}$, the Hankel function of the second kind. The last one suggests that we could search for the solution to our problem in the class of the Hankel functions.

Indeed, if we suppose $V_{m}(\chi) \equiv J_{m}(\chi) \pm \mathrm{i} Y_{m}(\chi)$, then Eq. $(7)$ can be reduced to much simpler equation of the form

$$
V_{m}=-\mathrm{i} \frac{2 \zeta}{\beta_{m}} V_{m-1}-\left[\mathcal{F}_{m}\left(\epsilon_{0}, \epsilon_{1}, \rho\right) / k \beta_{m} \cos \theta_{0}\right](\cos \nu \chi+\mathrm{i} \sin \nu \chi),
$$

where $\nu=(2 \zeta)^{-1} k \cos \theta_{0}$ and hence, $J_{m}(\chi)$ and $Y_{m}(\chi)$ should obey the following equations for $H_{m}^{(1)}$ :

$$
\begin{aligned}
& J_{m}(\chi)=\left(2 \zeta / \beta_{m}\right) Y_{m-1}(\chi)-\operatorname{Re}[f] \\
& Y_{m}(\chi)=-\left(2 \zeta / \beta_{m}\right) J_{m-1}(\chi)+\operatorname{Im}[f]
\end{aligned}
$$

and

$$
\begin{aligned}
& J_{m}(\chi)=-\left(2 \zeta / \beta_{m}\right) Y_{m-1}(\chi)-\operatorname{Re}[f], \\
& Y_{m}(\chi)=\left(2 \zeta / \beta_{m}\right) J_{m-1}(\chi)+\operatorname{Im}[f]
\end{aligned}
$$

for $H_{m}^{(2)}$. Here $\operatorname{Re}[f]$ and $\operatorname{Im}[f]$ stand for the real and imaginary parts of the complex-valued function $f$ :

$$
\begin{aligned}
& \operatorname{Re}[f]=\left[A \cos \left(k \cos \theta_{0} y\right)-B \sin \left(k \cos \theta_{0} y\right)\right]\left(\zeta / \beta_{m}\right), \\
& \operatorname{Im}[f]=\left[B \cos \left(k \cos \theta_{0} y\right)+A \sin \left(k \cos \theta_{0} y\right)\right]\left(\zeta / \beta_{m}\right),
\end{aligned}
$$

where 


$$
A \approx \frac{2 \pi k_{0}^{2} \rho_{1} \Omega}{\epsilon_{0}} \gamma\left(\frac{1}{\alpha_{1}}+\frac{1}{\alpha_{2}}\right), \quad B \approx \frac{\pi k_{0}^{2} \epsilon_{1} \rho_{1} \Omega}{\epsilon_{0}^{2}} \gamma\left(\frac{1}{\alpha_{3}}-\frac{1}{\alpha_{4}}\right)
$$

while $\gamma$ and $\alpha_{i}(i=1 \ldots 4)$ are defined in Appendix. Here we also took into account that $\Delta \epsilon \ll \epsilon_{0}$ and $\rho_{1}>\rho_{0}$. Each pair of these four equations (8), (9) can be considered as the nonlinear equations for determining $y$. At first sight this system of equations might appear to be overdetermined. Let us remember, however, that Eqs. (8) and (9) are not the systems of algebraic equations but the systems of transcendental ones. Most probable, they cannot be satisfied simultaneously for many $m$ 's and a single $y$ ("interaction length"), but perhaps, they can be satisfied approximately for some $m$ 's and different $y$ 's, each for its own $m$. This conjecture turns out to be valid, as it is seen in Figs. 2, 3, where some results of our calculations are shown.
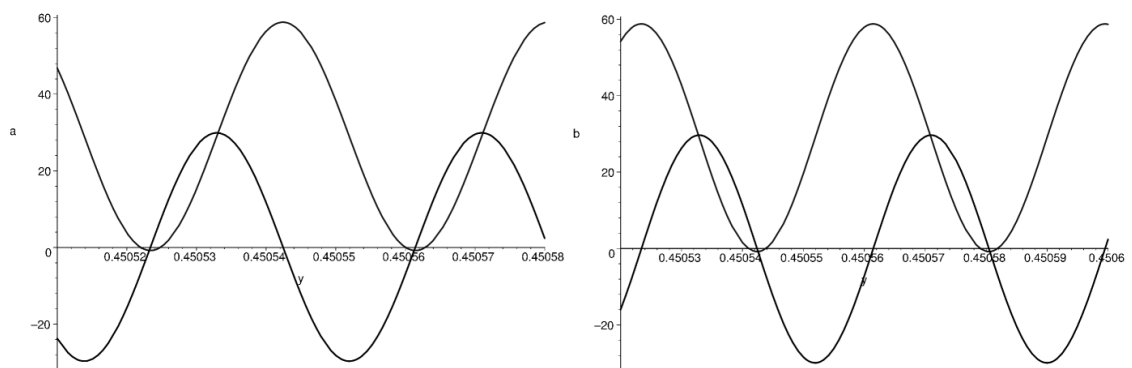

Fig. 2. (a) Numerical solution of Eq. (8) in the class of the Hankel functions of the first kind, $H_{m}^{(1)} ; m=2, \theta_{0}=0.01 \mathrm{rad}, y$ is given in $\mathrm{cm}$; (b) the same is for Eq. (9) (for the Hankel functions of the second kind), $H_{m}^{(2)} ; m$ and $\theta_{0}$ are as in the previous case.
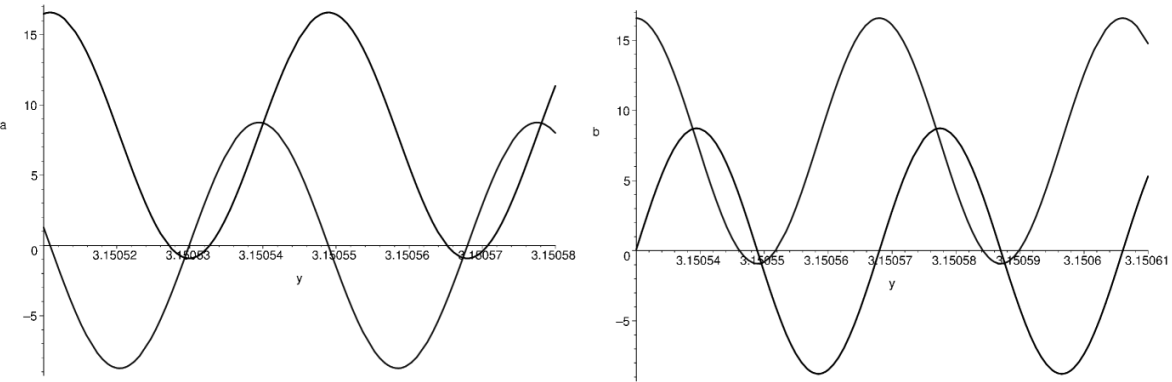

Fig. 3. (a) Solutions for $H_{m}^{(1)}, m=4, \theta=0.03 \mathrm{rad}$; (b) the same is for $H_{m}^{(2)}$.

In these figures the plotted curves represent the right-hand (or left-hand) sides of Eqs. (8), (9), if all the entries, except zeros, are in one side of the equation. Then the points of intersection of the curves with $y$-axis correspond to the solutions 
of each equation in a pair $((8)$ or $(9))$, that is, the value of "interaction length" at given $m$ and the incident angle $\theta_{0}$. Let us analyse at first Fig. 2a. It is clearly seen that these points do not coincide: the upper curve corresponds to the second of the two equations (8); it intersects $y$-axis at four points, two of them are in the vicinity of $y \approx 0.45052 \mathrm{~cm}$, while another two are near $y \approx 0.45056 \mathrm{~cm}$. The lower curve intersects $y$-axis at two points, each of them is just in between two intersection points of the upper curve near $y \approx 0.45052 \mathrm{~cm}$, as well as near $y \approx 0.45056 \mathrm{~cm}$. The similar results were obtained for the solutions of another pair of equations, Eqs. (9), that is, for the class of the Hankel functions of the second kind, $H_{m}^{(2)}$. They are shown in Fig. 2b. It is interesting to note that in all cases the absolute value of the diffracted beam amplitudes, $\left|V_{m}\right|$, practically (with the accuracy to 6 digits after decimal point) does not change in the whole range of changing of $y$ between the zeros of the first equation in each pair. The last one means that indeed, in this region two transcendental equations $((8)$ or $(9))$ can be satisfied simultaneously (but of course, approximately) for chosen values of $m$ and the incident angle $\theta_{0}$.

We should admit that if the distributed Gunn effect structure is concerned, we did not succeed in finding the solution to our problem for $m>2$ and for the reasonable values of $y$. "Reasonable" in this context means that $y$ should be smaller than $1 \mathrm{~cm}$. However, if we consider light diffraction by the plasmons in semiconductor films and set $y$ to be greater than $1 \mathrm{~cm}$, then we can find the solutions of Eq. (8) as well as Eq. (9) for greater $m$. Some of these results are presented in Fig. 3a,b. The calculations were done at the next values of the parameters involved: refractive index of GaAs was supposed to be 3.3, the equilibrium electron concentration $n_{0} \approx 10^{14} \mathrm{~cm}^{-3}$, peak value of electron concentration $n \approx 10^{16} \mathrm{~cm}^{-3}$, $\omega=1.5 \times 10^{15} \mathrm{~Hz}, \Omega=6-8 \times 10^{9} \mathrm{~Hz}$. Now we can comprehend in which sense this regime can be called "intermediate". It is intermediate between the Bragg and Raman-Nath diffractions, since first, $Q \approx 1$ (unlike the Bragg diffraction, when $Q>10$ and the Raman-Nath one, when $Q<1$ ), second, we have only one diffraction maximum for the given $L$ (just like in case of the Bragg diffraction), but unlike the Bragg regime, the diffraction order can be greater than unit just like in case of the Raman-Nath diffraction.

\section{Discussion and conclusion}

We have considered the possibility of light diffraction by the space charge waves which are the periodic domain trains induced at some circumstances in GaAs Gunn effect diode. This travelling charge wave can produce the modulation of semiconductor refractive index, since semiconductor susceptibility depends directly on the modulation of charge carriers density. Considering Gunn effect type device, we used at the calculations the typical values of the parameters: the width of the semiconductor sample to be $L_{x} \approx 10^{-3}-10^{-2} \mathrm{~cm}$, equilibrium electron concentration $n_{0} \approx 10^{14} \mathrm{~cm}^{-3}$, the electron concentration within the high field 
domain $n \approx 10^{16} \mathrm{~cm}^{-3}$. Assuming the condition $L_{y} \gg L_{x}$ to be fulfilled, that is, the condition which determines the distributed Gunn effect structure, we nevertheless suppose that "interaction length" is smaller than $1 \mathrm{~cm}$. According to our numerical simulations, the trends are the following: diminishing the angle at small values of $m$ allows to find simultaneous approximate solutions for the two equations in each pair ((8) or (9)) for the reasonable values of $y$, while increasing the angle does not. The same is valid for the diffraction order $m$ : the greater diffraction order is, the greater the "interaction length" becomes. Therefore, there exists some "optimum" concerning the diffraction order and the value of incident angle in case of the Gunn effect type device, since $L_{y}$ has to be within the reasonable limits of about a few tenths of $1 \mathrm{~cm}$. If one deals with SCW of the plasmon-type generated in semiconductor sample, one has more "degrees of freedom", that is, one can have greater $L_{y}$ and hence, greater $m$ and greater incident angle.

Estimating the efficiency of the deflector or directional coupler based on acoustic surface waves, one has to know what a fraction of the power of electric signal applied to the device is transformed into acoustic signal. In case of acoustic surface wave modulators this fraction is usually small and as a result, the refractive index modulation, being indirect, is also small. In case of the light diffraction by means of space charge waves we have all reasons to believe that such modulation, being direct, can be, first, greater and, second, could be achieved in much simpler and easier way.

In conclusion we would like to mention perhaps one of the most attractive possible applications of the device proposed in the paper. It is the guiding and deflecting of surface electromagnetic waves (SEW). SEW is simply a polarized wave propagating along the planar boundary which separates free space from the half space with a finite conductivity. SEW modes can occur for instance, near the infrared region in semiconductors such as GaP and GaAs [11] as well as on thin films of photonic band gap materials [12]. In semiconductors their behaviour can be controlled by doping, roughening of the surface, and external magnetic fields. Coupling the SEW device with that discussed in the paper could lead, perhaps to a kind of miniaturized photonic circuits combining waveguides, switchers, directional couplers, and so on.

\section{Appendix}

Here we show how Eq. (3) can be derived. Plugging (2) into (1), we have

$$
\begin{aligned}
\sum_{n=-\infty}^{\infty} & \left\{V_{n}^{\prime \prime}-2 \mathrm{i} k \cos \theta_{0} V_{n}^{\prime}(y)-\left[k^{2}+2 n k k_{0} \sin \theta_{0}+n^{2} k_{0}^{2}\right.\right. \\
& \left.\left.-(\omega+n \Omega)^{2} / c^{\prime 2}\right] V_{n}(y)+\left(\epsilon_{1} / \epsilon_{0} c^{\prime 2}\right)(\omega+n \Omega)^{2}\left[V_{n+1}(y)+V_{n-1}(y)\right]\right\} \\
& \times \exp \left(\mathrm{i}\left[(\omega+n \Omega) t-\left(k \sin \theta_{0}+n k_{0}\right) x-y k \cos \theta_{0}\right]\right) \equiv \mathcal{F}\left(\epsilon_{0}, \epsilon, \rho\right),
\end{aligned}
$$

where the primes stand for the corresponding derivatives with respect to $y$. 
Multiplying both sides of the equation by $\exp \left(-\mathrm{i}\left[(\omega+m \Omega) t+\left(k \sin \theta_{0}+m k_{0}\right) x+\right.\right.$ $\left.\left.y k \cos \theta_{0}\right]\right)$ and integrating over $x$ from $-\pi / k_{0}$ to $+\pi / k_{0}$ and over $t$ from $-\pi / \Omega$ to $+\pi / \Omega$, we get Eq. (3), where

$$
\begin{aligned}
& \mathcal{F}_{m}\left(\epsilon_{0}, \epsilon_{1}, \rho_{0}, \rho_{1}\right)=-\frac{2 \pi k_{0}}{\epsilon_{0}}\left[\left(\epsilon_{1} / \epsilon_{0}\right) \rho_{0}-\rho_{1}\right] \gamma\left(\frac{k_{0} \Omega}{\alpha_{1}}+\frac{k_{0} \Omega}{\alpha_{2}}\right) \\
& +\mathrm{i} \frac{\pi k_{0} \epsilon_{1} \rho_{1}}{\epsilon_{0}^{2}} \gamma\left(\frac{k_{0} \Omega}{\alpha_{3}}-\frac{k_{0} \Omega}{\alpha_{4}}\right), \\
& \alpha_{1}=[\omega+(m-1) \Omega]\left[k \sin \theta_{0}+(m-1) k_{0}\right], \\
& \alpha_{2}=[\omega+(m+1) \Omega]\left[k \sin \theta_{0}+(m+1) k_{0}\right], \\
& \alpha_{3}=[\omega+2(m-1) \Omega]\left[k \sin \theta_{0}+2(m-1) k_{0}\right], \\
& \alpha_{4}=[\omega+2(m+1) \Omega]\left[k \sin \theta_{0}+2(m+1) k_{0}\right], \\
& \gamma=\left[(-1)^{m+1} \cos \left(\frac{\pi \omega}{\Omega}\right)-1\right]\left[(-1)^{m+1} \cos \left(\frac{k \pi}{k_{0}} \sin \theta_{0}\right)-1\right] .
\end{aligned}
$$

\section{References}

[1] C.V. Brown, Em.E. Kriezis, S.J. Elston, J. Appl. Phys. 91, 3495 (2002).

[2] V.I. Berezhiani, S.M. Mahajan, arXiv:cond-mat/9604020 v1.

[3] H.W. Thim, J. Appl. Phys. 39, 3897 (1968).

[4] Yuo-Hsein Shiau, Phys. Rev. B 60, 15534 (1999-I).

[5] S.R. Elliott, The Physics and Chemistry of Solids, Chichester, West Sussex, Wiley, New York 1998.

[6] G.S. Simin, Fiz. Techn. Poluprovodn. (Sov. Phys. Techn. Semicond.) 10, 2025 (1976).

[7] M. Born, E. Wolf, Principles of Optics, Pergamon Press, Oxford 1970.

[8] M.G. Cohen, E.I. Gordon, Bell Syst Tech. J. 44, 693 (1965).

[9] R.W. Damon, W.T. Maloney, D.H. McMahon, in: Physical Acoustics, Vol. VIII, Eds. W.P. Mason, R.N. Thurston, Acad. Press, New York 1971.

[10] Handbook of Mathematical Functions with Formulas, Graphs and Mathematical Tables, Eds. M. Abramowitz, C.A. Stegun, Dover, New York 1972.

[11] S.S, Dhillon, J. Alton, S. Barbieri, S. Sirtori, A. de Rossi, M. Calligaro, H.E. Beere, D.A. Ritchie, in: Proc. 12th Conf. on Narrow Gap Semiconductors, Eds. J. Kono, J. Leotin, Taylor \& Francis, Paris 2006, p. 317.

[12] J.D. Joannopoulos, R.D. Meade, J.N. Winn, Photonic Crystals, Princeton University Press, 1995. 